\title{
Impact of SPARC expression on outcome in patients with advanced pancreatic cancer not receiving nab-paclitaxel: a pooled analysis from prospective clinical and translational trials
}

\begin{abstract}
Steffen Ormanns ${ }^{*}, 1,9$, Michael Haas ${ }^{2,9}$, Sibylle Baechmann ${ }^{1,2}$, Annelore Altendorf-Hofmann ${ }^{3}$, Anna Remold ${ }^{1,2}$, Detlef Quietzsch ${ }^{4}$, Michael R Clemens ${ }^{5}$, Martin Bentz ${ }^{6}$, Michael Geissler ${ }^{7}$, Helmut Lambertz ${ }^{8}$, Stephan Kruger ${ }^{2}$, Thomas Kirchner ${ }^{1}$, Volker Heinemann ${ }^{2}$ and Stefan Boeck ${ }^{\star}, 2$

${ }^{1}$ Institute of Pathology, Ludwig-Maximilians Universität München, Thalkirchner Strasse 36, D-80337 Munich, Germany; ${ }^{2}$ Department of Internal Medicine III and Comprehensive Cancer Center, Klinikum Grosshadern, Ludwig-Maximilians Universität München, Marchioninistrasse 15, D-81377 Munich, Germany; ${ }^{3}$ Department of Surgery, Jena University Hospital, Jena, Germany; ${ }^{4}$ Department of Internal Medicine II, Klinikum Chemnitz gGmbH, Chemnitz, Germany; ${ }^{5}$ Department of Hematology and Oncology, Mutterhaus der Boromaeerinnen, Trier, Germany; ${ }^{6}$ Department of Internal Medicine III, Klinikum Karlsruhe, Karlsruhe, Germany; ${ }^{7}$ Department of Gastroenterology and Oncology, Klinikum Esslingen, Esslingen, Germany and ${ }^{8}$ Department of Hematology and Oncology, Klinikum Garmisch-Partenkirchen, Garmisch-Partenkirchen, Germany
\end{abstract}

Background: Conflicting results on the role of secreted protein acidic and rich in cysteins (SPARC) expression have been reported in resected pancreatic ductal adenocarcinoma (PDAC), and its prognostic and/or predictive role in advanced PDAC (aPDAC) has not been extensively investigated yet. This study was designed to evaluate SPARC expression as a biomarker in aPDAC patients (pts) not receiving nab-paclitaxel.

Methods: Using immunohistochemistry, we examined the stromal as well as the tumoral (i.e., cytoplasmic) SPARC expression in tumour tissue (primary tumours and metastases) of 134 aPDAC pts participating in completed prospective clinical and biomarker trials. The SPARC expression levels were correlated to the pts' clinicopathological parameters and survival times.

Results: Sixty-seven per cent of the analysed tumours showed high stromal SPARC expression, which was not associated with overall survival (OS, median 9.1 vs 7.6 months, $P=0.316$ ). A positive cytoplasmic SPARC expression was detected in $55 \%$ of the tumours and correlated significantly with inferior progression-free survival (PFS, 6.2 vs 8.6 months, $P=0.004)$ and OS (7.8 vs 8.4 months, $P=0.032$ ). This association was strongest for pts, where primary tumour tissue was examined (PFS: 6.7 vs 10.8 months, $P=0.004$; OS: 7.9 vs 11.9 months, $P=0.030$ ), whereas no significant correlation was detected for pts, where only metastatic tissue was available (PFS: 5.8 vs 6.6 months, $P=0.502$; OS: 7.0 vs 7.8 months, $P=0.452$ ). In pts receiving gemcitabine-based chemotherapy cytoplasmic SPARC expression was significantly associated with an inferior PFS and OS (PFS: 6.2 vs 9.2 months, $P=0.002$; OS 7.3 vs 9.9 months, $P=0.012$ ), whereas no such association was detected for stromal SPARC expression or for pts receiving fluoropyrimidine-based chemotherapy.

Conclusion: We identified cytoplasmic SPARC expression in the primary tumour as a biomarker associated with inferior PFS and OS in aPDAC. Cytoplasmic SPARC expression may furthermore act as a negative predictive biomarker in pts treated with gemcitabine-based chemotherapy.

\footnotetext{
*Correspondence: Dr S Ormanns; E-mail: steffen.ormanns@med.unimuenchen.de or Dr S Boeck; E-mail: stefan.boeck@med.unimuenchen.de ${ }^{9}$ These authors contributed equally to this work.
}

Received 19 August 2016; accepted 4 October 2016; published online 1 November 2016

(c) 2016 Cancer Research UK. All rights reserved 0007-0920/16 
Despite extensive efforts in basic and clinical research in the last decades, pancreatic ductal adenocarcinoma (PDAC) is still a devastating disease, with mortality rates almost equal to incidence rates (Malvezzi et al, 2014). About $80 \%$ of PDAC patients (pts) are diagnosed in inoperable, often metastatic stages of disease with palliative chemotherapy being the only remaining therapeutic option (Heinemann et al, 2012a). In addition, the overall PDAC incidence rates are rising in western societies (Rahib et al, 2014), which furthermore stresses the need for an intensified search for effective therapies as well as prognostic and predictive biomarkers-as these are still lacking, especially in advanced, that is metastatic disease (Kruger et al, 2014). PDAC often displays a dense desmoplastic stroma, which has been associated with chemoresistance (Von Hoff et al, 2009; Sinn et al, 2014 b) as it has been shown to inhibit drug penetration as a physical barrier and due to high tissue pressures (Provenzano et al, 2012). This desmoplastic stroma is composed of lymphocytes, leukocytes and cancer-associated fibroblasts, which produce an extracellular matrix consisting of proteins such as collagens, hyaluronic acids and other proteins (Mahadevan and Von Hoff, 2007). Among them, the calcium-binding protein secreted protein acidic and rich in cysteine (SPARC) has recently gained increasing attention as it interacts with and modulates the extracellular matrix and influences cell migration as well as angiogenesis and tissue remodelling (Nagaraju et al, 2014). It is overexpressed in PDAC compared with normal pancreatic tissue and increases the invasiveness of PDAC cells (Guweidhi et al, 2005). The peritumoral expression of the stromal component SPARC (also known as osteonectin-1, ON-1) has been linked to a worse prognosis in previous studies of resected PDAC, whereas the data on the impact of tumoral SPARC expression on $\mathrm{pt}$ outcome are conflicting in this context (Infante et al, 2007; Sinn et al, 2014b). Surprisingly, stromal SPARC expression has been shown to be associated with improved treatment response to nanoparticle albumin-bound paclitaxel (nab-paclitaxel)-treated aPDAC pts enrolled within an early phase I/II study, resulting in increased survival (Von Hoff et al, 2011). This effect was attributed to the affinity of SPARC to binding albumin, resulting in a depletion of tumoral stroma and an improved chemotherapy delivery to the tumour cells (Von Hoff et al, 2011). In fact, similar results have been obtained in genetically engineered mouse models of PDAC and smaller pts studies using nab-paclitaxel (Alvarez et al, 2013), although there seems to be limited effects of SPARC expression on nab-paclitaxel efficacy in mice (Neesse et al, 2014). In contrast, a recently published post hoc analysis of the pivotal phase III MPACT trial comparing nab-paclitaxel plus gemcitabine $v s$ gemcitabine alone did not show a significant effect of SPARC expression on overall survival (OS) or on nab-paclitaxel efficacy in metastatic PDAC pts (Hidalgo et al, 2015).

As the majority of the previously published studies mainly focused on resected PDAC pts, the prognostic significance of SPARC expression in the peritumoral stroma and in the tumour cells has not been extensively studied in advanced PDAC (aPDAC) yet. We thus examined the association of tumoral SPARC expression and its expression in the peritumoral stroma with clinicopathological parameters and survival times in a well-defined aPDAC population treated with various regimens of non-nabpaclitaxel-containing palliative first-line chemotherapy.

\section{PATIENTS AND METHODS}

PDAC pt population. Samples of 134 aPDAC pts were collected retrospectively from pts treated within three terminated German multicentre chemotherapy trials (Heinemann et al, 2006; Boeck et al, 2008; Heinemann et al, 2012b), as well as from a prospective single-centre biomarker study performed at the Comprehensive Cancer Center of Ludwig-Maximilians-University in Munich $\left(\mathrm{CCC}^{\mathrm{LMU}}\right)$. Patients treated within the AIO-PK0104 phase III study were only included in the current analysis if they were enrolled before amendment 1 (active from March 2006 on) that added erlotinib to first-line chemotherapy with either gemcitabine or capecitabine (Boeck et al, 2010). Clinical data were retrieved from the participating hospitals and the studies databases. Only pts receiving conventional cytotoxic chemotherapy were included into the current SPARC biomarker study, excluding pts treated with targeted agents (such as erlotinib) or nab-paclitaxel. The OS times were calculated from the start of palliative first-line chemotherapy to death from any cause. This retrospective biomarker study was approved by the local ethics committee of Ludwig-MaximiliansUniversity of Munich (approval number 554-11). All pts included in the clinical studies gave written informed consent before any study-specific procedure was performed; pts included in our local prospective biomarker CCC $^{\mathrm{LMU}}$ database provided informed consent for the use of their clinical data, blood and archival tumour tissue for scientific research.

Tumour samples, SPARC immunohistochemistry and scoring algorithm. We retrieved formalin-fixed paraffin-embedded archival tumour tissue of 134 aPDAC pts irrespective of the tissue origin (primary or metastatic, resection or biopsy). To examine the tumoral and stromal SPARC expression using immunohistochemistry (IHC) on $4 \mu \mathrm{m}$ whole-mount tissue sections, a Ventana Benchmark XT autostainer was used (Ventana, Tucson, AZ, USA). Briefly, the slides were dewaxed and antigenicity was retrieved using the Ventana antigen retrieval solution CC1 ( $\mathrm{pH}$ 8.4, Ventana) for $30 \mathrm{~min}$. The slides were then incubated with the mouse monoclonal SPARC antibody (1:800 dilution, clone ON-1, Invitrogen, Carlsbad, CA, USA) for $32 \mathrm{~min}$, and after secondary antibody incubation (Ultraview Kit; Ventana) the staining was visualised using diaminobenzidine (Ventana). Appropriate positive (human placenta) and negative control tissue (human skeletal muscle) were included in each staining run (Supplementary Figure S1).

The intensity of SPARC expression in the peritumoral stroma was assessed using a two-tier scoring system according to Illei et al (2013) by two experienced pathologists (SO and TK) blinded to the pts' clinical outcome. Briefly, absent or weak SPARC staining in $<25 \%$ of the stroma was classified as low expression (low), while moderate to strong SPARC staining in over $25 \%$ of the tumour stroma was considered as high expression (high). Equally, cytoplasmic SPARC expression in the carcinoma cells was assessed, defining an absent or faint and speckled cytoplasmic staining in $<10 \%$ of the tumour cells as absent expression and thus as SPARC-negative case. In contrast, a clearly detectable weak, moderate or strong cytoplasmic staining intensity in over $10 \%$ of the tumour cells was considered a SPARC-positive case. Discrepant cases were discussed until agreement was reached. The results were correlated with the pts previously determined clinicopathological parameters and survival times (progression-free survival (PFS) and OS) from the beginning of palliative chemotherapy on.

Statistical analyses. The Kaplan-Meier method with log-rank tests and Cox regression were used for univariate survival analyses. For multivariate analysis a stepwise forward Cox regression was applied. The relation of SPARC expression and its relation with pts clinicopathological characteristics were evaluated using $\chi^{2}$-tests.

\section{RESULTS}

Patient characteristics and clinicopathological variables. A total of 107 of the 134 included pts were treated within prospective 
clinical trials (e.g., 58 pts (Heinemann et al, 2006), 29 pts (Boeck et al, 2008) and 15 pts (Heinemann et al, 2012b)) and 27 pts were derived from an ongoing prospective PDAC biomarker study conducted at the CCC ${ }^{\mathrm{LMU}}$. Median age within the study population (79 male and 55 female) was 62 years. Eighty-three pts had a Karnofsky performance status (KPS) $>80 \%$, which was associated with an improved OS (6.7 vs 10.6 months; hazard ratio (HR) 1.537, 95\% confidence interval (CI) 1.051-2.249, $P=0.025$; Table 1; Supplementary Figure S2A) but not PFS $(P=0.062$, Supplementary Figure S2B). Fourteen pts had locally advanced pancreatic cancer (LAPC) and 120 pts metastatic disease at chemotherapy initiation, with the latter being associated with shorter OS and PFS from the beginning of palliative chemotherapy on (OS 8.0 vs 12.0 months, HR 1.705, 95\% CI 0.891-3.265, $P=0.103$; PFS 10.0 vs 6.8 months, HR 1.795 , 95\% CI $0.864-3.729$, $P=0.111$; Table 1; Supplementary Figure S2C and D), though not statistically significant (potentially due to the small sample size of LAPC pts). In all, 112 pts received gemcitabine-based first-line treatment and an additional 22 pts fluoropyrimidine-based chemotherapy, respectively, with no significant difference in OS (8.3 vs 7.2 months; HR 1.552, 95\% CI 0.970-2.482, $P=0.064$; Supplementary Figure S2E); however, PFS was longer in pts treated with a gemcitabine-containing first-line regimen (7.4 vs 4.0 months, HR 2.111, 95\% CI 1.226-3.635, $P=0.006$; Supplementary Figure S2F). A total of 14 pts had no apparent metastasis upon treatment initation (LAPC pts), 84 pts had a single metastatic site and 36 pts had more than one metastatic site, which was not significantly associated with shorter PFS or OS (for details see Table 1 as well as Supplementary Figure S2G and H). Median OS calculated from the initiation of palliative chemotherapy of the entire study cohort was 8.3 months (95\% CI 7.05-9.51). Neither pt age group ( $<60$ years $v s \geqslant 60$ years) nor sex or tumour grading was associated with PFS or OS (Table 1).

Stromal and cytoplasmic SPARC expression. Stromal and cytoplasmic SPARC expression was examined in 134 samples, with available corresponding clinicopathological data. Archival tissue origin was primary tumour (PT) in 64 cases (including 45 resected specimens) and metastasis (M) in 70 pts. SPARC expression in the desmoplastic peritumoral stroma was heterogeneously distributed, with an accentuated expression in the

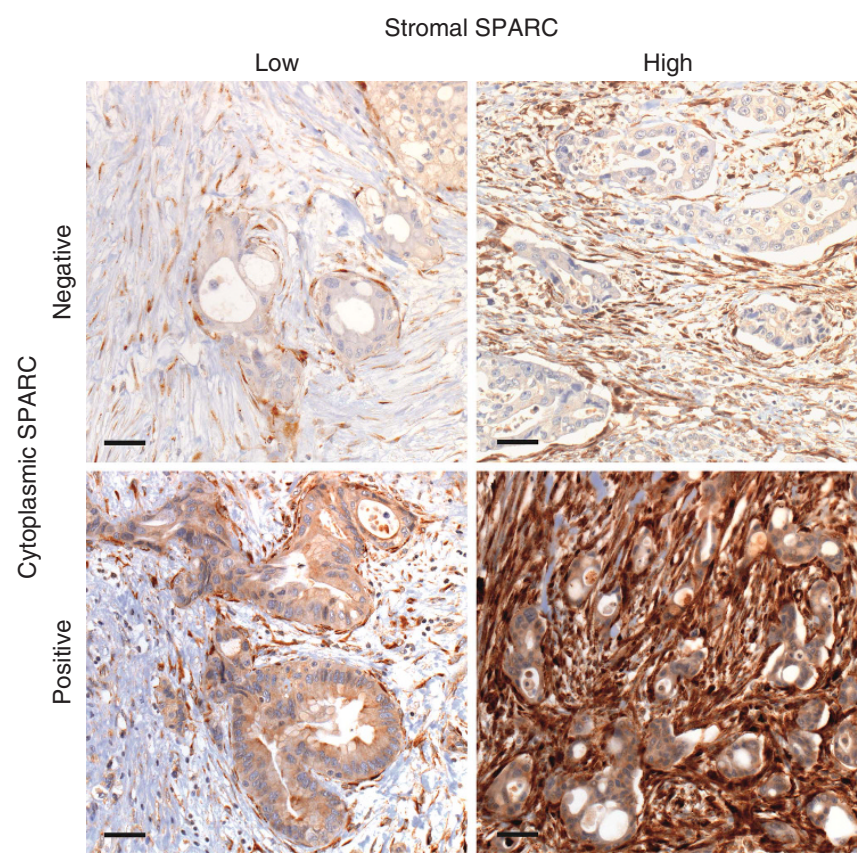

Figure 1. Variable stromal and cytoplasmic SPARC expression in PDAC tissue. Immunohistochemical staining of SPARC (ON-1) in exemplary PDAC tissue shows variable expression in the peritumoral stroma and tumoral cytoplasm. Magnification $=\times 200$. Scale bars indicate $50 \mu \mathrm{m}$.

Table 1. Frequency of the patients' clinicopathological characteristics (sex, age, KPS, disease stage at start of first-line chemotherapy, type of first-line chemotherapy, tumour grade and number of metastatic sites) and correlation with corresponding overall and progression-free survival

\begin{tabular}{|c|c|c|c|c|c|c|c|c|c|}
\hline & $n$ & OS (months) & $P$ (log-rank) & HR & $95 \% \mathrm{Cl}$ & PFS (months) & $P$ (log-rank) & HR & $95 \% \mathrm{Cl}$ \\
\hline \multicolumn{10}{|l|}{ Sex } \\
\hline Male & 79 & 8.3 & \multirow[t]{2}{*}{0.852} & \multirow[t]{2}{*}{0.966} & \multirow[t]{2}{*}{$0.668-1.395$} & 7.0 & \multirow[t]{2}{*}{0.681} & \multirow[t]{2}{*}{1.094} & \multirow[t]{2}{*}{$0.712-1.681$} \\
\hline Female & 55 & 7.3 & & & & 4.7 & & & \\
\hline \multicolumn{10}{|l|}{ Age group } \\
\hline$<60$ years & 54 & 8.3 & \multirow[t]{2}{*}{0.348} & \multirow[t]{2}{*}{1193} & \multirow[t]{2}{*}{$0.825-1.725$} & 6.8 & \multirow[t]{2}{*}{0.682} & \multirow[t]{2}{*}{1.094} & \multirow[t]{2}{*}{$0.712-1.681$} \\
\hline$\geqslant 60$ years & 80 & 7.9 & & & & 7.0 & & & \\
\hline$>80$ & 83 & 10.6 & 0.025 & 1537 & $1.051-2.249$ & 7.6 & 0.062 & & $0.976-2.332$ \\
\hline \multicolumn{10}{|c|}{ Stage at start of palliative CTX } \\
\hline Locally advanced & 14 & 12.0 & \multirow[t]{2}{*}{0.103} & \multirow[t]{2}{*}{1705} & \multirow[t]{2}{*}{$0.89-3.265$} & 10.0 & \multirow[t]{2}{*}{0.111} & 1.795 & \multirow[t]{2}{*}{$0.864-3.729$} \\
\hline Metastatic & 120 & 8.0 & & & & 6.8 & & & \\
\hline \multicolumn{10}{|c|}{ First-line CTX, CTX type } \\
\hline Gemcitabine-based & 112 & 8.3 & 0.064 & 1552 & $0.970-2.482$ & 7.4 & 0.006 & 2.111 & $1.226-3.635$ \\
\hline G3-G4 & 80 & 7.9 & & & & 6.6 & & & \\
\hline Metastatic sites & & & & & & & & & \\
\hline 0 & 14 & 12.0 & 0.220 & 1281 & $0.945-1.737$ & 10.0 & 0.084 & 1,509 & $1.049-2.170$ \\
\hline 1 & 84 & 8.3 & & & & 7.0 & & & \\
\hline$>1$ & 36 & 7.3 & & & & 5.5 & & & \\
\hline
\end{tabular}


stromal tissue close to carcinoma cells (Figure 1). Of 134 samples, $90(67 \%)$ showed a high and $44(33 \%)$ a low expression of SPARC in the peritumoral stroma. Cytoplasmic SPARC expression was homogenously distributed throughout the carcinoma cells, though much weaker than in the adjacent desmoplastic stroma (Figure 1). Of 134 samples, 74 (55\%) showed a measurable positive cytoplasmic SPARC expression, whereas 60 (45\%) were classified as SPARC negative. No statistically significant correlation between stromal and cytoplasmic SPARC expression was detected $(P=0.268$; Supplementary Table S1).

SPARC expression, clinicopathological variables and survival analyses. First, we examined the association of the possibly confounding clinicopathological variables sex, age group, disease stage, KPS, tissue origin, tumour grading and number of metastatic sites within the stromal as well as the cytoplasmic SPARC expression subgroup and detected no statistically significant interactions, besides a higher number of cytoplasmic SPARCpositive cases in pts with a KPS $\leqslant 80$ (67\% vs $44 \%, P=0.010$; Supplementary Table S2). Overall, high stromal SPARC expression was not associated with PFS (7.0 vs 5.9 months; HR 1.254, 95\% CI $0.805-1.953, P=0.314$ ) nor with OS (9.1 vs 7.6 months; HR 0.823, 95\% CI 0.562-1.205, $P=0.316$; Table 2; Figure 2A and B). We obtained similar results for stromal SPARC in the PT and the M subgroup (PT subgroup: 10.8 vs 8.3 months, $P=0.857 ; \mathrm{M}$ subgroup: 7.8 vs 6.8 months, $P=0.319$; Table 2; Supplementary Figure S3A and $\mathrm{B}$ ). In contrast, we detected a statistically significant inferior PFS and OS for pts showing a positive cytoplasmic SPARC expression (PFS: 6.2 vs 8.6 months, HR 1.878, 95\% CI 1.208-2.921, $P=0.0045$; OS: 7.8 vs 8.4 months, HR 1.505, 95\% CI 1.034-2.190, $P=0.032$; Figure 2C and D). This effect in fact was restricted to the PT subgroup (PFS: 6.7 vs 10.8 months, $P=0.004$; OS: 7.9 vs 11.9 months, $P=0.030$; Figure $2 \mathrm{E}$ and $F$ ), as we found no significant difference in our survival analyses between cytoplasmic SPARC-positive and cytoplasmic SPARC-negative cases in the M subgroup (PFS: 5.8 vs 6.6 months, $P=0.502$; $\quad$ OS: 7.0 vs 7.8 months, $P=0.452$; Table 2; Supplementary Figure S3C and D).

In order to test whether the stromal or cytoplasmic SPARC expression could additionally interact with the efficacy of the applied chemotherapy, we exploratively compared PFS and OS according to SPARC expression levels in the first-line gemcitabinebased treated $(n=112)$ and in the first-line fluoropyrimidinebased treated $(n=22)$ pt populations; this of note was done as an unplanned subgroup analysis. A positive cytoplasmic SPARC expression was significantly associated with inferior PFS and OS in the gemcitabine-treated group (PFS: 6.2 vs 9.2 months, $P=0.002$; OS: 7.3 vs 9.9 months, $P=0.012$; Table 2; Figure $3 \mathrm{~A}$ and $\mathrm{B}$ ), whereas no such association was detected within the smaller fluoropyrimidine-treated subgroup (PFS: 6.7 vs 2.2 months, $P=0.032$; OS: 9.1 vs 4.1 months, $P=0.106$; Figure $3 \mathrm{C}$ and $\mathrm{D}$ ). In contrast, there rather was a trend for a better OS and a longer PFS in fluoropyrimidine-treated pts with a positive cytoplasmic SPARC expression. We found no differences in PFS or OS

Table 2. Correlation of overall and progression-free survival (Kaplan-Meier estimates, log-rank tests and Cox regression) according to stromal or cytoplasmic SPARC expression in the total study population, in the primary tumour and metastatic tissue subgroups and in the gemcitabine-based and fluoropyrimidine- based treatment subgroups

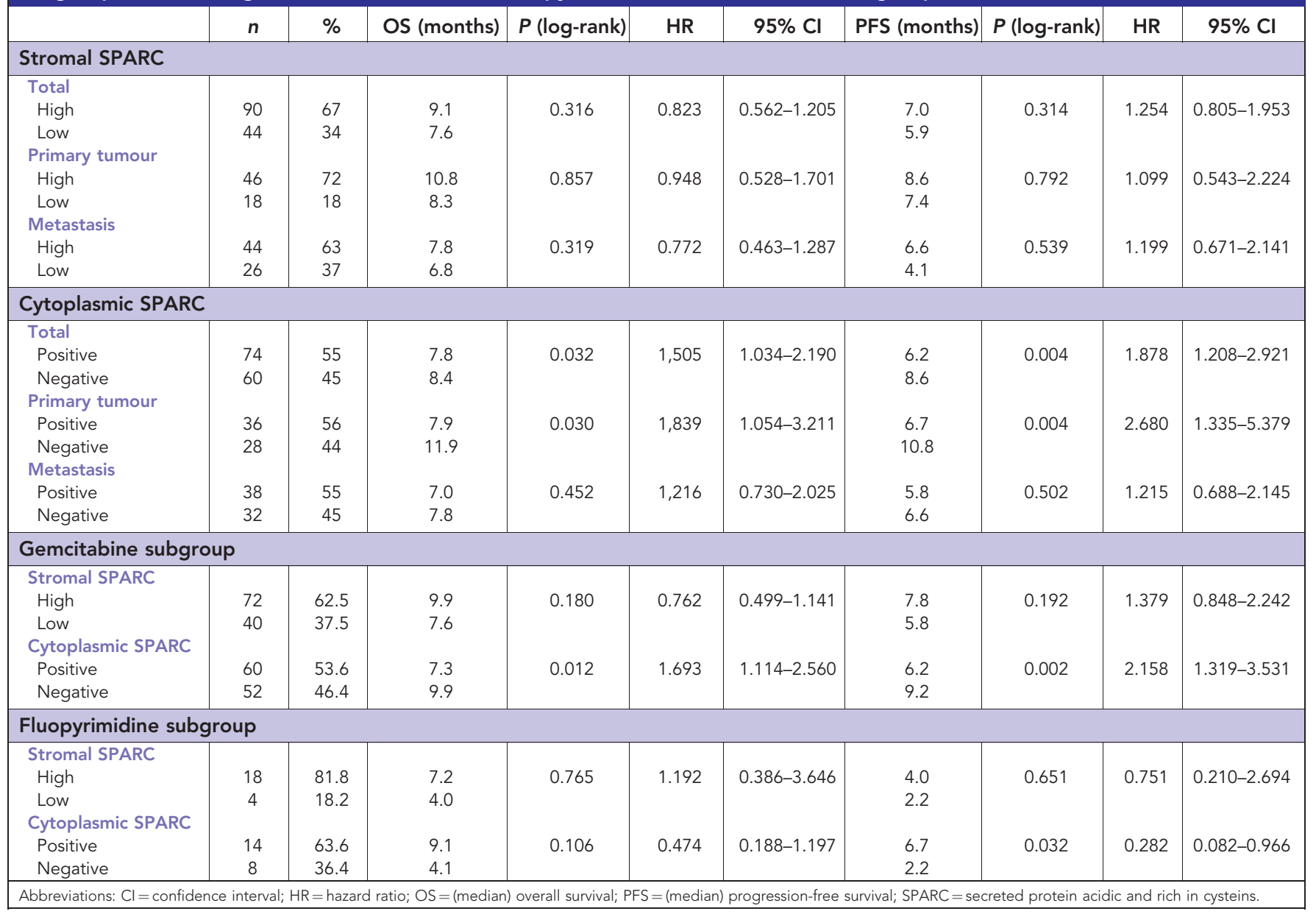


according to stromal SPARC expression neither in the gemcitabine- nor in the fluoropyrimidine-treated subgroups (Table 2; Supplementary Figure S4A-D).

Within stepwise forward multivariate Cox regression analyses adjusting for KPS, tumour grading, number of metastatic sites, stage of disease and cytoplasmic SPARC expression, KPS group was the only independent prognostic factor for OS (HR 1.483, 95\%CI 1.012-2.174, $P=0.043)$, whereas cytoplasmic SPARC expression remained borderline significant (HR 1.454, 95\%CI $0.977-2.212, P=0.052)$. Interestingly, cytoplasmic SPARC expression was an independent factor for a poorer PFS in the overall study population (HR 1.878, $P=0.005)$ and also for both PFS (HR 2.262, $P=0.001)$ and $\mathrm{OS}(\mathrm{HR} 1.689, P=0.014)$ in gemcitabine-treated pts (Supplementary Table S3).

\section{DISCUSSION}

To our knowledge, this is one of the first biomarker studies examining the peritumoral and cytoplasmic SPARC expression in aPDAC pts that did not receive nab-paclitaxel containing palliative chemotherapy. Using IHC, we were able to show that SPARC expression in PDAC tissue can reliably be assessed in resected tumour samples, as well as in biopsies independently from the tissue origin, that is, primary or metastatic tumours. In line with previously published studies (Sinn et al, 2014b; Gundewar et al, $2015)$, we were able to show in our well-defined study cohort $(80 \%$ of the included pts were treated within prospective clinical trials) that a high SPARC expression in the peritumoral stroma occurs in the majority of PDAC tumours, but had no association with PFS or OS in none of the studied subgroups (Table 2). In contrast, cytoplasmic SPARC expression was associated with an inferior PFS and $O S$ in the gemcitabine-treated subgroup and also in the overall study population when PT samples were examined. Additional multivariate analyses confirmed cytoplasmic SPARC expression as an independent factor for PFS and OS in gemcitabine-treated pts.

Several studies on SPARC expression in PDAC - using different detection systems and scoring methods - have been published so far, overall resulting in controversial data (Prenzel et al, 2006; Infante et al, 2007; Mantoni et al, 2008; Miyoshi et al, 2010; Von Hoff et al, 2011; Sinn et al, 2014b; Gundewar et al, 2015; Hidalgo et al, 2015; Table 3). Concerning stromal SPARC expression, our findings in aPDAC are in contrast to previously published studies in resected PDAC, demonstrating inferior OS and a lack of gemcitabine efficacy in pts with high stromal SPARC expression (Infante et al, 2007; Sinn et al, 2014b), as well as increased tumour progression (Gundewar et al, 2015). In a translational analysis from the adjuvant CONKO-001 study, the prognostic effect of peritumoral SPARC expression was limited to pts treated with adjuvant gemcitabine, supporting the idea of stromal SPARC as a potential negative predictive biomarker for adjuvant chemotherapy (Sinn et al, 2014b). This observation may be due to increased SPARC expression in dense desmoplastic stroma and is in line with the hypothesis of the peritumoral stroma as a treatment barrier in PDAC (Provenzano et al, 2012). Accordingly, studies have identified stromal SPARC expression as a treatment target of nabpaclitaxel-based chemotherapy (Von Hoff et al, 2011; Alvarez et al, 2013), thus reducing the desmoplastic stroma and offering a
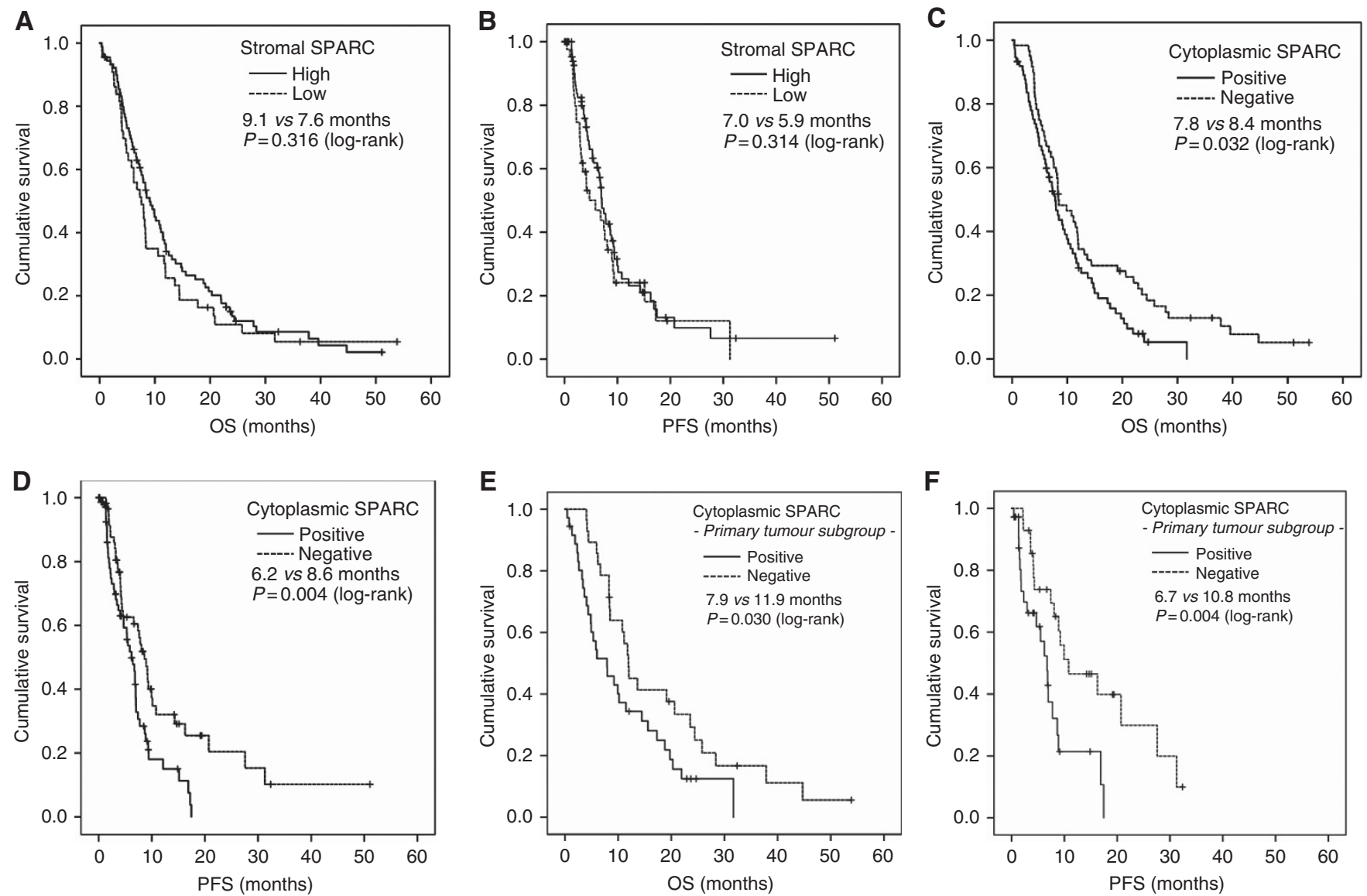

Figure 2. Cytoplasmic but not stromal SPARC expression correlates with inferior overall survival (A, C and E) and progression-free survival (B, D and F) of aPDAC patients. Univariate analysis (Kaplan-Meier curves and log-rank tests) of (A and B) stromal and (C and D) cytoplasmic SPARC expression as a biomarker in the tumour tissue of aPDAC pts treated with palliative, non-nab-paclitaxel containing chemotherapy; cytoplasmic SPARC expression in the ( $E$ and $\mathbf{F})$ primary tumour subgroup. Crossed lines indicate censored cases. 

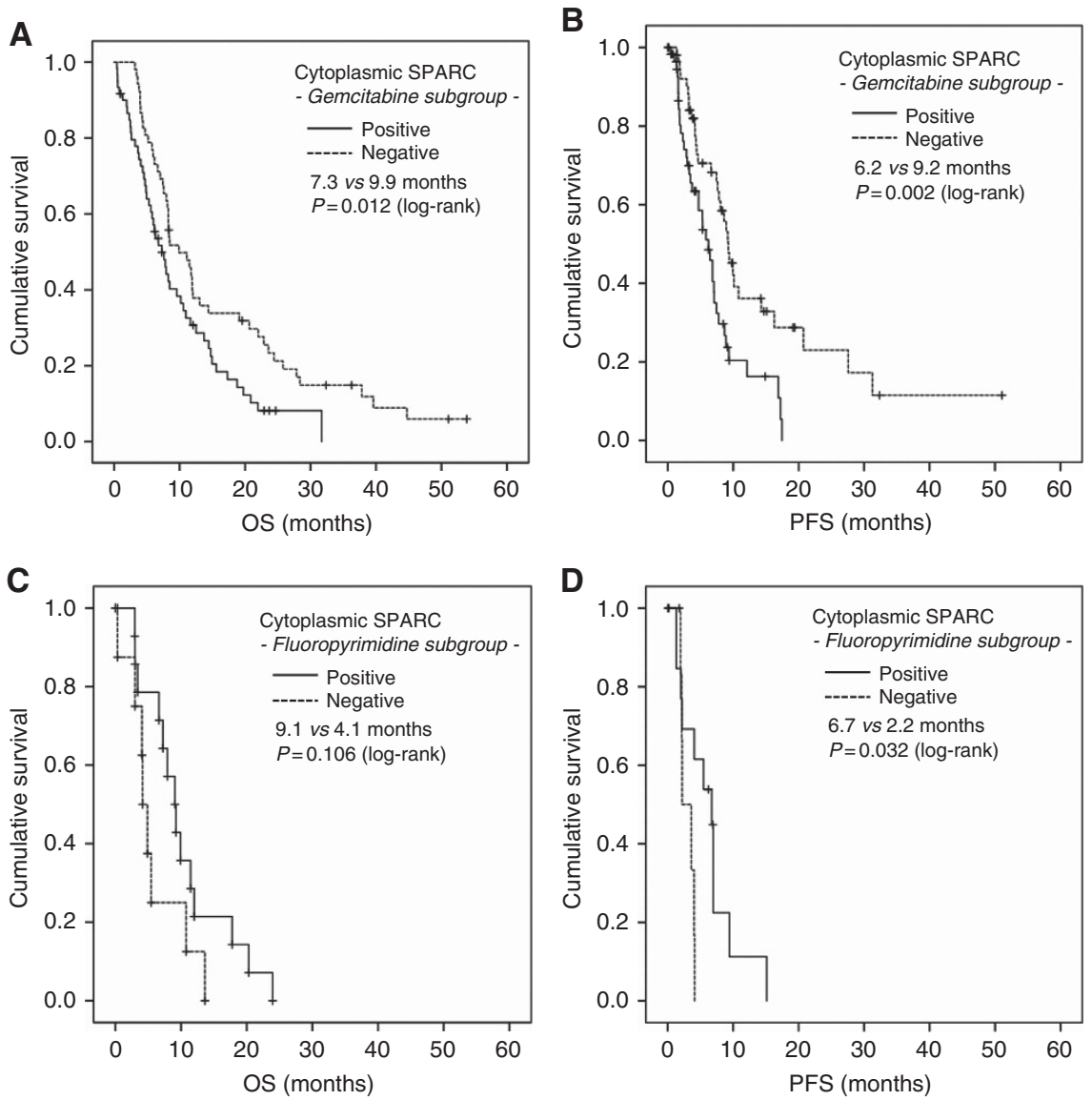

Figure 3. Cytoplasmic SPARC expression as a potential negative predictive biomarker for overall survival ( $\mathbf{A}$ and $\mathbf{C})$ and progression-free survival (B and D) in gemcitabine-treated aPDAC patients. Univariate analysis (Kaplan-Meier curves and log-rank tests) of cytoplasmic SPARC expression in the tumour tissue of ( $\mathbf{A}$ and $\mathbf{B}$ ) gemcitabine-based treated aPDAC pts and (C and $\mathbf{D}$ ) fluoropyrimidine-based treated aPDAC pts. Crossed lines indicate censored cases.

survival advantage for PDAC pts. However, the idea of the peritumoral stroma as a mere treatment barrier in PDAC may be too simplistic as it does not reflect the complex signalling interaction between the stromal and the epithelial compartment. Indeed, activated stromal cells have been shown to have a tumour-promoting effect (Moffitt et al, 2015), resulting in decreased survival, which can be overcome by stromal modulation (Sherman et al, 2014). On the other hand, recent preclinical data showed a deleterious effect of myofibroblast depletion in genetically engineered mouse models of PDAC, resulting in poor tumour differentiation, increased aggressiveness, proliferation, vascularisation and immunosuppression, finally leading to inferior OS (Özdemir et al, 2014; Rhim et al, 2014). Moreover, as the data on the prognostic role of the peritumoral stroma in humans (as measured by $\alpha$-SMA expression) are still conflicting (Özdemir et al, 2014; Sinn et al, 2014a) and clinical trials for stromal depletion in PDAC have failed (Bramhall et al, 2002; Moore et al, 2003) clear conclusions on this subject cannot be drawn yet. Especially, the exact functions and interactions of each stromal component, such as myofibroblasts, extracellular matrix, pancreatic stellate cells and immune cells in tumour promotion or inhibition are contradictory, context dependent and still poorly understood in its complexity (Neesse et al, 2015).

Data from the large phase III MPACT study recently confirmed an OS benefit for the combination of nab-paclitaxel plus gemcitabine compared with gemcitabine alone (Von Hoff et al, 2013; Hidalgo et al, 2015). Thus, nab-paclitaxel plus gemcitabine has evolved as a new standard treatment option in pts with metastatic PDAC (Borazanci and Von Hoff, 2014). However, a predictive role of high stromal SPARC expression for nabpaclitaxel efficacy could not be confirmed in a detailed translational analysis from MPACT (Hidalgo et al, 2015). This recently published post hoc sub-study ( $n=301$ out of 861$)$ reported much lower rates of high stromal and notably of high tumoral SPARC expression and did not show a significant effect of stromal or tumoral SPARC expression on OS in both chemotherapy subgroups and the overall study population (Hidalgo et al, 2015). In our study reported here, we used the same antibody (ON-1 (Invitrogen)) as the MPACT investigators, and the assessment of stromal SPARC expression was done by a previously reported and validated scoring system (Illei et al, 2013). In line with the findings from MPACT, we did not detect a significant negative association of high stromal SPARC expression and PFS or OS. Indeed, high stromal SPARC expression was even associated with a trend towards an improved OS, though not statistically significant (9.1 vs 7.6 months, $P=0.316$; Table 2). The high rate of cytoplasmic SPARC positivity in our study and its association with a worse PFS and OS are in stark contrast to the translational MPACT results (Hidalgo et al, 2015), which is most likely due to a much more sensitive cutoff for SPARC positivity used in our study. In fact, using the same stringent cutoff as the MPACT investigators, we would have obtained a similarly low percentage of cytoplasmic SPARC expression (2.3\%), which was - as expected - not associated with OS $(P=0.411)$.

The conflicting results on stromal and cytoplasmic SPARC as a prognostic or predictive biomarker in PDAC may partially be based on different methodology concerning protein detection and staining evaluation but also on the origin of the tumour samples 


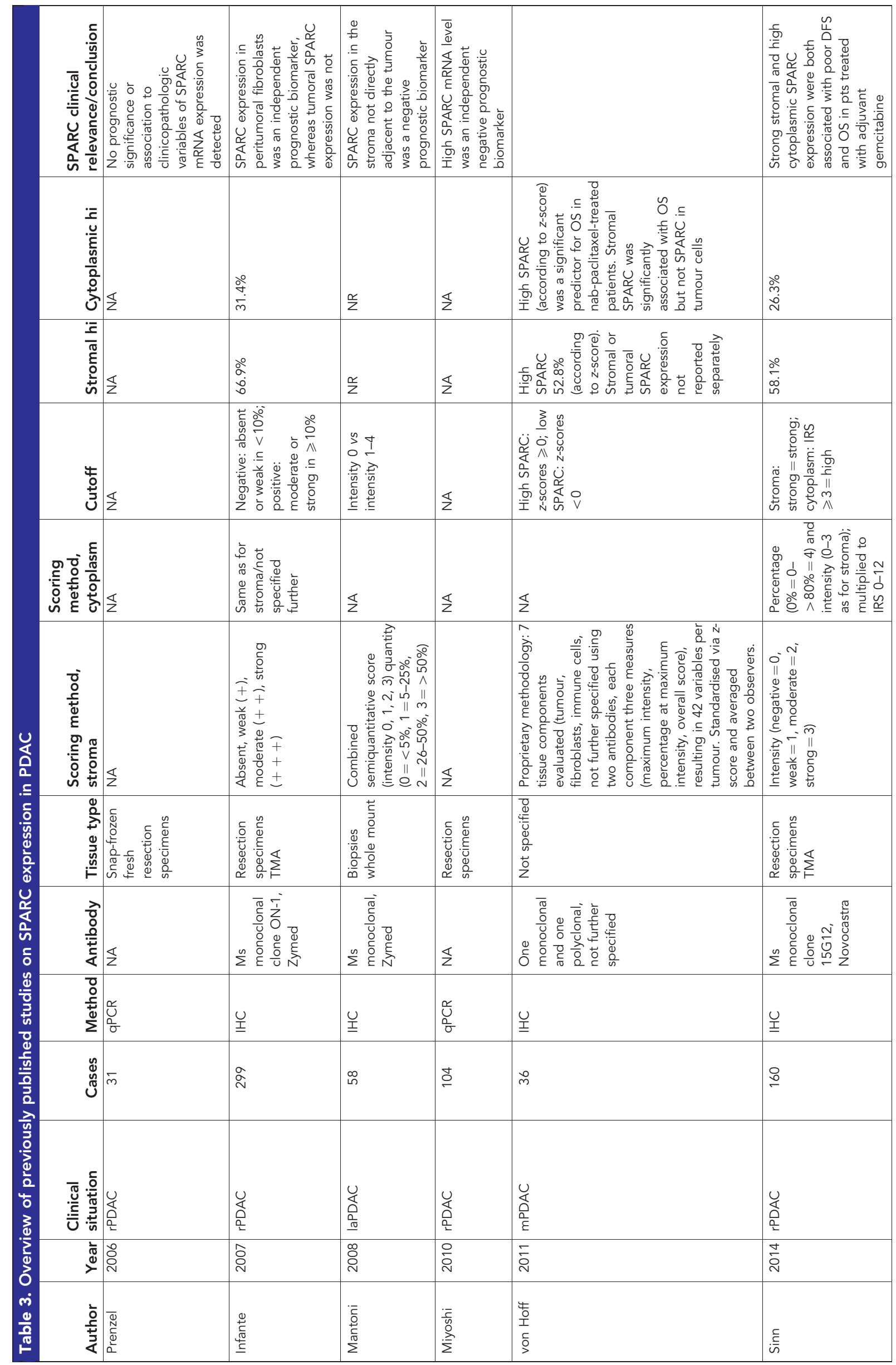




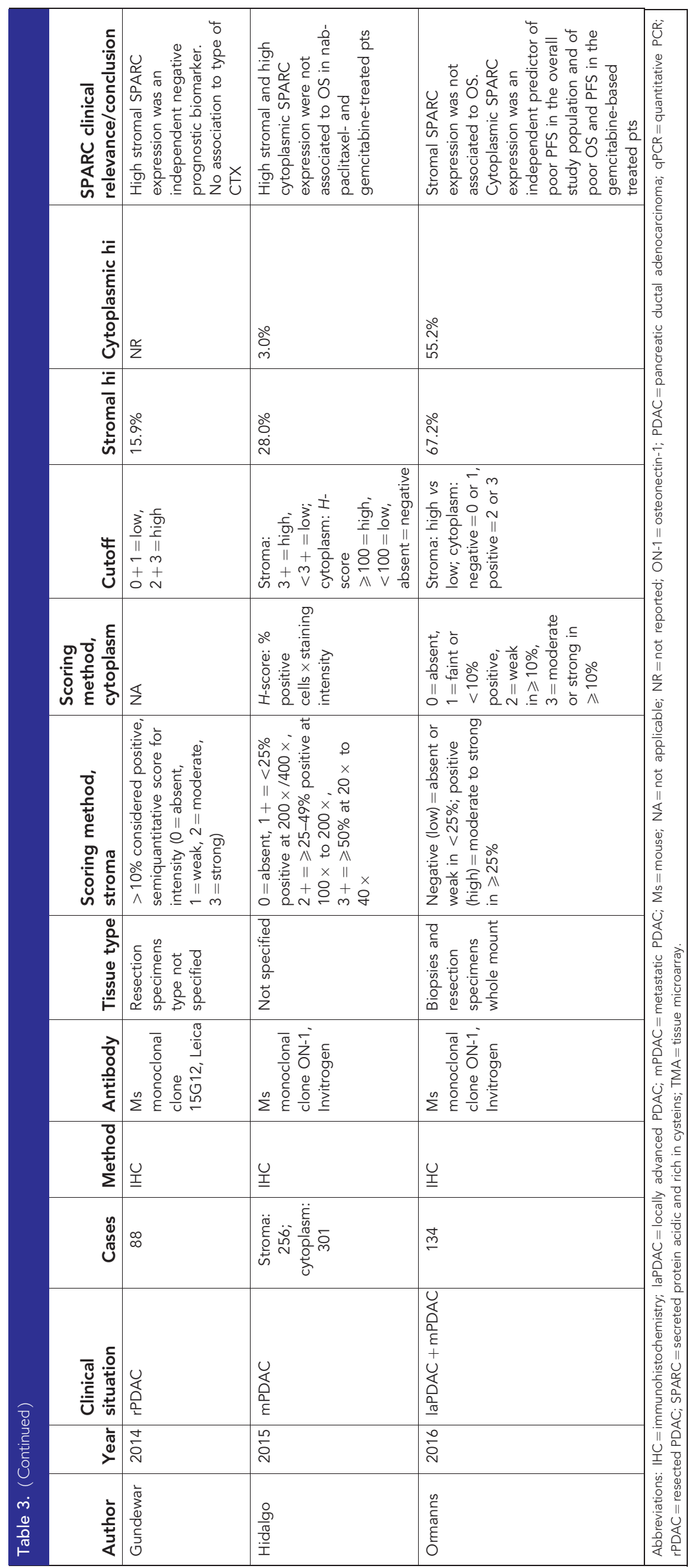


examined (Table 3). Notably, in the translational MPACT study primarily tissue samples from metastatic lesions or unidentified origin were used, whereas only $11 \%$ were confirmed pancreatic lesions. In contrast, all the studies confirming SPARC either as prognostic (Infante et al, 2007; Gundewar et al, 2015) and/or predictive (Sinn et al, 2014b) biomarker, were performed on resected primary PDAC tumours. Differential SPARC expression in primary and metastatic tissue has been reported (Guweidhi et al, 2005), which we could not confirm in the present study. Using both PT samples $(n=70)$ as well as metastatic tissue $(n=64)$, we detected nearly identical rates of stromal and cytoplasmic SPARC expression in both subgroups. Notably, a prognostic impact of cytoplasmic SPARC expression was detected in the PT subgroup only. A main limitation for this preliminary observation may be based on the fact that within our PT subgroup both tumour tissues from the pancreatic primary of pts with metachronous (that previously underwent surgical pancreatic resection) and of pts with synchronous metastatic disease were included. This novel clinical finding, however, could be in line with a functional role for cytoplasmic SPARC in pre-metastatic tumour progression, as SPARC pre-clinically has been shown to increase the invasive properties of PDAC tumour cells (Guweidhi et al, 2005; Seux et al, 2011), possibly via induction of matrix metalloproteases or modulation of their activity (Vaz et al, 2015).

In addition, we observed (within an explorative subgroup analysis) a potential survival benefit of a low or absent cytoplasmic SPARC expression for pts receiving gemcitabine-based treatment, whereas no such significant association was found for the smaller group of fluoropyrimidine-based treated pts. In the latter subgroup there even was a trend towards improved PFS and OS for pts with cytoplasmic SPARC-expressing tumours. However, the statistical power in the fluoropyrimidine subgroup was clearly limited due to the rather small sample size $(n=22)$. One thus might at least hypothesise a negative predictive role of cytoplasmic SPARC expression for gemcitabine efficacy; if that observation could be confirmed by other groups, a possible biomarker for a treatment decision between gemcitabine- or fluoropyrimidine-based first-line regimens according to cytoplasmic SPARC expression in aPDAC pts could perhaps be obtained.

Its retrospective nature, the heterogeneous chemotherapy regimens applied, as well as the use of both biopsy material and resection specimens can be considered as the main limitations of our translational biomarker study. However, these characteristics may at least in part also be interpreted as strength of the current study, as our findings hold true for a broad range of clinically variable aPDAC pts that reflect daily clinical practice. Specifically, the important observation that the kind of tissue origin (primary $v s$ metastatic) may have a significant impact on the value of a biomarker in PDAC is of high relevance for future biomarker trials in this disease. In this context, investigators should also keep in mind that tumour tissue from the pancreatic primary may perform differently in biomarker studies based on the time point of collection (e.g., archival tissue from a previous surgery in curative intent $v$ s biopsy of the primary in a pt with synchronous metastatic disease). Thus, the need for repeated biopsies in future PADC biomarker studies arises in order to generate the best translational evidence possible.

Taken together, our data and previously published studies (Infante et al, 2007; Gundewar et al, 2015) indicate that stromal SPARC expression in PDAC may serve as a prognostic biomarker only in primarily resected PDAC, whereas no such association can be found in the metastatic PDAC situation. In contrast, our findings show that cytoplasmic SPARC expression may serve as biomarker associated with inferior PFS and $O S$ in non-nab-paclitaxel-treated aPDAC pts (with this effect being restricted to the PT subgroup) and may furthermore potentially also act as a negative predictive biomarker for the efficacy of gemcitabine-based chemotherapy in aPDAC. An external validation of these hypothesis-generating results is necessary and strongly recommended; ideally, such a confirmatory biomarker study should of course be performed within a prospective clinical trial. Specifically, the role of tumoral/ cytoplasmatic SPARC expression in PT specimens and its impact on treatment outcome should for example be re-investigated within the global APACT trial (that has already completed recruitment), which compares adjuvant gemcitabine $v s$ gemcitabine plus nab-paclitaxel for 6 months after curative-intent resection of PDAC (NCT01964430).

\section{ACKNOWLEDGEMENTS}

SO is supported by a grant from the Friedrich-Baur-Stiftung, Munich. This study was funded by an unrestricted research grant from Celgene, Germany.

\section{CONFLICT OF INTEREST}

The authors declare no conflict of interest.

\section{REFERENCES}

Alvarez R, Musteanu M, Garcia-Garcia E, Lopez-Casas P, Megias D, Guerra C, Muñoz M, Quijano Y, Cubillo A, Rodriguez-Pascual J (2013) Stromal disrupting effects of nab-paclitaxel in pancreatic cancer. $\mathrm{Br}$ J Cancer 109(4): 926-933.

Boeck S, Hoehler T, Seipelt G, Mahlberg R, Wein A, Hochhaus A, Boeck H-P, Schmid B, Kettner E, Stauch M (2008) Capecitabine plus oxaliplatin (CapOx) versus capecitabine plus gemcitabine (CapGem) versus gemcitabine plus oxaliplatin $(\mathrm{mGemOx})$ : final results of a multicenter randomized phase II trial in advanced pancreatic cancer. Ann Oncol 19(2): 340-347.

Boeck S, Vehling-Kaiser U, Waldschmidt D, Kettner E, Märten A, Winkelmann C, Klein S, Kojouharoff G, Gauler T, von Weikersthal LF (2010) Erlotinib $150 \mathrm{mg}$ daily plus chemotherapy in advanced pancreatic cancer: an interim safety analysis of a multicenter, randomized, cross-over phase III trial of the 'Arbeitsgemeinschaft Internistische Onkologie'. Anticancer Drugs 21(1): 94-100.

Borazanci E, Von Hoff DD (2014) Nab-paclitaxel and gemcitabine for the treatment of patients with metastatic pancreatic cancer. Expert Rev Gastroenterol Hepatol 8(7): 739-747.

Bramhall S, Schulz J, Nemunaitis J, Brown P, Baillet M, Buckels J (2002) A double-blind placebo-controlled, randomised study comparing gemcitabine and marimastat with gemcitabine and placebo as first line therapy in patients with advanced pancreatic cancer. Br J Cancer 87(2): 161-167.

Gundewar C, Sasor A, Hilmersson KS, Andersson R, Ansari D (2015) The role of SPARC expression in pancreatic cancer progression and patient survival. Scand J Gastroenterol 50(9): 1170-1174.

Guweidhi A, Kleeff J, Adwan H, Giese NA, Wente MN, Giese T, Büchler MW, Berger MR, Friess H (2005) Osteonectin influences growth and invasion of pancreatic cancer cells. Ann Surg 242(2): 224.

Heinemann V, Haas M, Boeck S (2012a) Systemic treatment of advanced pancreatic cancer. Cancer Treat Rev 38(7): 843-853.

Heinemann V, Quietzsch D, Gieseler F, Gonnermann M, Schönekäs H, Rost A, Neuhaus H, Haag C, Clemens M, Heinrich B (2006) Randomized phase III trial of gemcitabine plus cisplatin compared with gemcitabine alone in advanced pancreatic cancer. J Clin Oncol 24(24): 3946-3952.

Heinemann V, Vehling-Kaiser U, Waldschmidt D, Kettner E, Märten A, Winkelmann C, Klein S, Kojouharoff G, Gauler TC, von Weikersthal LF (2012b) Gemcitabine plus erlotinib followed by capecitabine versus capecitabine plus erlotinib followed by gemcitabine in advanced pancreatic cancer: final results of a randomised phase 3 trial of the 'Arbeitsgemeinschaft Internistische Onkologie'(AIO-PK0104). Gut 62: 751-759. 
Hidalgo M, Plaza C, Musteanu M, Illei P, Brachmann CB, Heise C, Pierce D, Lopez-Casas PP, Menendez C, Tabernero J (2015) SPARC expression did not predict efficacy of nab-paclitaxel plus gemcitabine or gemcitabine alone for metastatic pancreatic cancer in an exploratory analysis of the phase III MPACT trial. Clin Cancer Res 21(21): 4811-4818.

Illei PB, Conde E, Dominguez N, Plaza C, Redondo P, Suarez-Gauthier A, Heise C, Hidalgo M, Lopez-Rios F (2013) SPARC expression in pancreatic adenocarcinoma: development of a robust, predictive immunohistochemical assay and scoring method. In Laboratory Investigation. Vol. 93, pp 425A-425A. Nature Publishing Group: New York, NY, USA.

Infante JR, Matsubayashi H, Sato N, Tonascia J, Klein AP, Riall TA, Yeo C, Iacobuzio-Donahue C, Goggins M (2007) Peritumoral fibroblast SPARC expression and patient outcome with resectable pancreatic adenocarcinoma. J Clin Oncol 25(3): 319-325.

Kruger S, Haas M, Ormanns S, Bächmann S, Siveke JT, Kirchner T, Heinemann V, Boeck S (2014) Translational research in pancreatic ductal adenocarcinoma: current evidence and future concepts. World $J$ Gastroenterol 20(31): 10769.

Mahadevan D, Von Hoff DD (2007) Tumor-stroma interactions in pancreatic ductal adenocarcinoma. Mol Cancer Ther 6(4): 1186-1197.

Malvezzi M, Bertuccio P, Levi F, La Vecchia C, Negri E (2014) European cancer mortality predictions for the year 2014. Ann Oncol 25(8): 1650-1656.

Mantoni TS, Schendel RR, Rödel F, Niedobitek G, Al-Assar O, Masamune A, Brunner TB (2008) Stromal SPARC expression and patient survival after chemoradiation for non-resectable pancreatic adenocarcinoma. Cancer Biol Ther 7(11): 1806-1815.

Miyoshi K, Sato N, Ohuchida K, Mizumoto K, Tanaka M (2010) SPARC mRNA expression as a prognostic marker for pancreatic adenocarcinoma patients. Anticancer Res 30(3): 867-871.

Moffitt RA, Marayati R, Flate EL, Volmar KE, Loeza SGH, Hoadley KA, Rashid NU, Williams LA, Eaton SC, Chung AH (2015) Virtual microdissection identifies distinct tumor-and stroma-specific subtypes of pancreatic ductal adenocarcinoma. Nat Genet 47(10): 1168-1178.

Moore M, Hamm J, Dancey J, Eisenberg P, Dagenais M, Fields A, Hagan K, Greenberg B, Colwell B, Zee B (2003) Comparison of gemcitabine versus the matrix metalloproteinase inhibitor BAY 12-9566 in patients with advanced or metastatic adenocarcinoma of the pancreas: a phase III trial of the National Cancer Institute of Canada Clinical Trials Group. J Clin Oncol 21(17): 3296-3302.

Nagaraju GP, Dontula R, El-Rayes BF, Lakka SS (2014) Molecular mechanisms underlying the divergent roles of SPARC in human carcinogenesis. Carcinogenesis 35(5): 967-973.

Neesse A, Algül H, Tuveson DA, Gress TM (2015) Stromal biology and therapy in pancreatic cancer: a changing paradigm. Gut 64: 1476-1484.

Neesse A, Frese KK, Chan DS, Bapiro TE, Howat WJ, Richards FM, Ellenrieder V, Jodrell DI, Tuveson DA (2014) SPARC independent drug delivery and antitumour effects of nab-paclitaxel in genetically engineered mice. Gut 63(6): 974-983.

Özdemir BC, Pentcheva-Hoang T, Carstens JL, Zheng X, Wu C-C, Simpson TR, Laklai H, Sugimoto H, Kahlert C, Novitskiy SV (2014) Depletion of carcinoma- associated fibroblasts and fibrosis induces immunosuppression and accelerates pancreas cancer with reduced survival. Cancer Cell 25(6): 719-734.

Prenzel KL, Warnecke-Eberz U, Xi H, Brabender J, Baldus SE, Bollschweiler E, Gutschow CA, Holscher A, Schneider PM (2006) Significant overexpression of SPARC/osteonectin mRNA in pancreatic cancer compared to cancer of the papilla of Vater. Oncol Rep 15(5): 1397.

Provenzano PP, Cuevas C, Chang AE, Goel VK, Von Hoff DD, Hingorani SR (2012) Enzymatic targeting of the stroma ablates physical barriers to treatment of pancreatic ductal adenocarcinoma. Cancer Cell 21(3): 418-429.

Rahib L, Smith BD, Aizenberg R, Rosenzweig AB, Fleshman JM, Matrisian LM (2014) Projecting cancer incidence and deaths to 2030: the unexpected burden of thyroid, liver, and pancreas cancers in the United States. Cancer Res 74(11): 2913-2921.

Rhim AD, Oberstein PE, Thomas DH, Mirek ET, Palermo CF, Sastra SA, Dekleva EN, Saunders T, Becerra CP, Tattersall IW (2014) Stromal elements act to restrain, rather than support, pancreatic ductal adenocarcinoma. Cancer Cell 25(6): 735-747.

Seux M, Peuget S, Montero M, Siret C, Rigot V, Clerc P, Gigoux V, Pellegrino E, Pouyet L, N'guessan P (2011) TP53INP1 decreases pancreatic cancer cell migration by regulating SPARC expression. Oncogene 30(27): 3049-3061.

Sherman MH, Ruth TY, Engle DD, Ding N, Atkins AR, Tiriac H, Collisson EA, Connor F, Van Dyke T, Kozlov S (2014) Vitamin D receptor-mediated stromal reprogramming suppresses pancreatitis and enhances pancreatic cancer therapy. Cell 159(1): 80-93.

Sinn M, Denkert C, Striefler J, Pelzer U, Stieler J, Bahra M, Lohneis P, Dörken B, Oettle H, Riess H (2014a) $\alpha$-Smooth muscle actin expression and desmoplastic stromal reaction in pancreatic cancer: results from the CONKO001 study. Br J Cancer 111(10): 1917-1923.

Sinn M, Sinn B, Striefler J, Lindner J, Stieler J, Lohneis P, Bischoff S, Bläker H, Pelzer U, Bahra M (2014b) SPARC expression in resected pancreatic cancer patients treated with gemcitabine: results from the CONKO-001 study. Ann Oncol 25(5): 1025-1032.

Vaz J, Ansari D, Sasor A, Andersson R (2015) SPARC: a potential prognostic and therapeutic target in pancreatic cancer. Pancreas 44(7): 1024.

Von Hoff DD, Ervin T, Arena FP, Chiorean EG, Infante J, Moore M, Seay T, Tjulandin SA, Ma WW, Saleh MN (2013) Increased survival in pancreatic cancer with nab-paclitaxel plus gemcitabine. New Engl J Med 369(18): 1691-1703.

Von Hoff DD, Korn R, Mousses S (2009) Pancreatic cancer-could it be that simple? A different context of vulnerability. Cancer Cell 16(1): 7-8.

Von Hoff DD, Ramanathan RK, Borad MJ, Laheru DA, Smith LS, Wood TE, Korn RL, Desai N, Trieu V, Iglesias JL (2011) Gemcitabine plus nabpaclitaxel is an active regimen in patients with advanced pancreatic cancer: a phase I/II trial. J Clin Oncol 29(34): 4548-4554.

This work is published under the standard license to publish agreement. After 12 months the work will become freely available and the license terms will switch to a Creative Commons AttributionNonCommercial-Share Alike 4.0 Unported License.

Supplementary Information accompanies this paper on British Journal of Cancer website (http://www.nature.com/bjc) 